\title{
Managing control programs for ovine caseous lymphadenitis and paratuberculosis in Australia, and the need for persistent vaccination
}

This article was published in the following Dove Press journal:

Veterinary Medicine: Research and Reports

24 March 2014

Number of times this article has been viewed

\section{Peter Andrew Windsor \\ Faculty of Veterinary Science, University of Sydney, Camden, NSW, Australia}

Correspondence: Peter A Windsor Faculty of Veterinary Science, University of Sydney, 410 Werombi Road, Camden, NSW 2570, Australia

$\mathrm{Tel}+61293511610$

Fax +612 935। 1618

Email peter.windsor@sydney.edu.au
Abstract: Ovine caseous lymphadenitis (CLA) and ovine Johne's disease (OJD) or paratuberculosis have been serious diseases in the Australian sheep industry, mainly causing losses from abattoir condemnations from CLA or mortalities on the farm from OJD. CLA is now a disease of minimal concern, with clinical cases reported rarely. Although OJD continues to spread through parts of the sheep population, the catastrophic losses in flocks occurring prior to the introduction of vaccination are now uncommon. Change-management factors relevant to the improvements in both prevalence and producer concerns for CLA and OJD were examined, including drivers and motivation for change, resistance to change, knowledge management, farming system dimensions and leadership. Although extension programs addressing disease risk factors are likely to be of relevance to improved knowledge and attitudes towards disease risk management of producers, improvements in disease-control practices were considered largely attributable to the introduction of vaccination programs for CLA in 1983 and OJD in 2002. Inclusion of the CLA antigen within clostridial vaccines ("6 in 1" vaccine) enabled routine annual CLA vaccination to occur in an increasing proportion of the national flock, with estimates of CLA prevalence suggesting a decline from $26 \%$ in 1995 to $5.2 \%$ in 2009 . Encouraging the routine vaccination of lambs for OJD (Gudair vaccine) in infected flocks to reduce or avoid losses significantly reduced the within-flock prevaccination-postvaccination median prevalence from $2.72 \%$ to $0.72 \%$, based on estimated shedding rates of Mycobacterium avium subsp. paratuberculosis determined by pooled fecal culture in 37 infected flocks vaccinating for at least 5 years. Although persistent use of CLA vaccine is a convenient intervention for producers, promoting the persistent use of OJD vaccination to continue disease suppression when clinical cases are undetectable, plus improvements in biosecurity, remain a challenge for animal-health authorities. Despite concerns of vaccine efficacy and safety issues with OJD vaccination, persistent vaccination has produced a profound improvement in the health of Australian sheep, and is a positive development of relevance to sheep production in other countries.

Keywords: CLA, caseous lymphadenitis, OJD, ovine Johne's disease, paratuberculosis, sheep health, vaccination, disease-control programs

\section{Introduction}

Ovine caseous lymphadenitis (CLA) caused by Corynebacterium pseudotuberculosis (CPTB) and ovine Johne's disease (OJD) or paratuberculosis caused by Mycobacterium avium subsp. paratuberculosis (MAP; mostly involving the S strain in Australia), are chronic systemic bacterial diseases of near-global distribution and importance. ${ }^{1,2}$ Although caused by very different pathogens, both CLA and OJD involve chronic inflammatory lesions of the lymphoid system in their pathogenesis. The presence of sheep with subclinical disease is important in maintaining both infections in flocks, 
and persistent use of vaccination that invokes both humoral and cell-mediated immune responses and suppresses disease expression is critical to their control.

In CLA, CPTB enters the live animal via skin wounds with release of the exotoxin phospholipase D (PLD) and a mycolic acid surface lipid (although other virulence factors have been proposed), leading to dermal necrosis with inflammation and increased vascular permeability. This promotes the invasiveness of the organism with transport to the regional lymph nodes through phagocytes, causing soft caseous lesions of lymphadenitis that have been described as abscesses. ${ }^{1}$ The inflammatory response may prevent infection from progressing beyond the cutaneous lesion, but usually local lymphadenitis occurs, followed by destruction of the lymph node, and unless these lesions fistulate externally, they usually progress to chronic granulomatous lymphadenitis, forming so-called cheesy gland lesions that are more correctly described as granulomas. CLA may then disseminate to the abdominal and thoracic viscera, where similar granulomatous lesions may occur, with the mediastinal lymph nodes responsible for dissemination of the infection following fistulation into the bronchi, forming an aerosol that readily transmits to other sheep, especially in close confinement and when skin lesions occur, as in shearing. ${ }^{1}$

In OJD, MAP mostly infects the live animal via the fecal-oral route, entering via the intestinal tract and then the lymphatic system, where it resides in $\mathrm{M}$ cells overlying Peyer's patches in the ileum, although prenatal infection is now well described. ${ }^{3,4}$ Chronic granulomatous enteritis and lymphadenitis, particularly involving the mesenteric lymph nodes, develop when epithelioid cells containing numerous MAP accumulate in the lamina propria and submucosa, causing so-called multibacillary lesions, although in some animals so-called paucibacillary lesions occur where the MAP population is far less numerous.

\section{Significance of CLA and OJD in Australia}

Although the Australian sheep population in Australia has declined in recent years, it was estimated there were 74.7 million sheep on 43,760 properties in mid-2011.5 CLA was probably introduced to Australia with the earliest arrivals of sheep over 200 years ago. It has been a disease of considerable economic importance, with estimated losses in 1991-1992 at AU\$30-40 million per annum, largely from the cost of carcass condemnations to prevent unsightly lesions from appearing in retail sheep-meat products, particularly in exported live sheep or products. ${ }^{1}$
OJD was most likely introduced into Australia much more recently, with the initial diagnosis made in the state of New South Wales (NSW) in the early 1980s. ${ }^{6}$ It caused sporadic problems until the mid-1990s, when the current epidemic of the disease emerged. For a period, OJD became the most important endemic disease of Australian sheep, due to extraordinary losses on farms in some areas, even exceeding 20\% per annum (personal observations), and escalating costs of disease control that aimed to restrict the spread of the disease to uninfected regions and states. ${ }^{6}$ A study of 12 farms in 2003 identified that the average OJD mortality rate was $7.8 \%$ (range $1.8 \%-14.6 \%$ ), causing an average decrease in gross margin due to OJD infection of $8.5 \%$ (range $3.1 \%-15.8 \%$ ) per farm, with CLA occurring rarely on these farms. ${ }^{7,8}$

Commercially available vaccines for CLA (Glanvac ${ }^{\circledR} 6$, then "6 in 1"; Zoetis, West Ryde, Australia) and OJD (Gudair ${ }^{\circledR}$; Zoetis) became available for producer use in Australia in 1984 and 2002, respectively. ${ }^{1,6}$ CLA vaccination has proved to be convenient to use regularly, as it is included in a combination preparation with other vaccines and is relatively cheap, safe, and easy to deliver, although it requires multiple doses to achieve protection and an annual booster to retain efficacy. Gudair vaccine is relatively inconvenient to use, because it is expensive, requires another intervention applied either at lamb marking or weaning, and although only a single dose is required for lifelong protection, it is an oil-based preparation and so more difficult to deliver by injection, increasing the risk of adverse reactions in sheep and self-injection by farmers or contractors. ${ }^{6}$

\section{Change in prevalence of CLA and OJD}

CLA has gradually become a disease of minimal concern to Australian sheep producers, with clinical cases reported rarely. However, surveys in 1995 identified that the disease was extremely widespread and present on $97 \%$ of farms in NSW, 91\% in Victoria, and 88\% in Western Australia, although the average estimated prevalence of CLA in the adult sheep population has been declining, and was estimated at 26\%. ${ }^{1,9}$ Routine abattoir surveillance introduced in 2006 to monitor the prevalence of OJD found $17 \%$ of 3,608 consignments of sheep to NSW abattoirs in that year having sheep with CLA, but only $1.3 \%$ of all sheep were found with CLA lesions. Prevalence data from abattoir surveillance indicate a decline in CLA prevalence in the national flock, from the 1995 estimate of $26 \%$ to $5.2 \%$ in $2009 .{ }^{9,10}$ Although variation in the sensitivity of abattoir surveillance between abattoirs and states is to be expected, with concerns 
of poor accuracy of the inspection technique, ${ }^{11}$ and reported variation in competence for OJD detection at slaughter, ${ }^{12}$ this is unlikely to account for the considerable variation in CLA prevalence between states in Australia. Estimates of CLA prevalence derived at slaughter of 1,604,659 sheep in 2009 were $12.9 \%$ in Victoria, $12.8 \%$ in Tasmania, 9.5\% in South Australia, 5.3\% in NSW, 4.8\% in Queensland, and $1.0 \%$ in Western Australia. ${ }^{10}$ Prolonged pathological surveillance over 10 years of the "tail of the mob" (sheep with low exercise tolerance that fall behind when the mob is moved) on a CLA-infected farm that had been vaccinating diligently for over a decade found that CLA cases with chronic granulomatous bronchial lymphadenitis (so-called shedders) occurred at between $0.5 \%$ and $1.5 \%$ of sheep that were well below flock-average condition score at necropsy (personal observations).

Although OJD has continued to spread to new areas of the Australian sheep population and remains a significant concern for sheep producers unfamiliar with managing the disease, the catastrophic losses that occurred in many flocks prior to the introduction of vaccination just over a decade $\mathrm{ago}^{7,8}$ are now rarely reported. OJD is no longer the "clinical" disease of major concern in the majority of those flocks that have been vaccinated for several years, although subclinical disease remains an important issue for OJD management. ${ }^{6}$ Notwithstanding the concerns about accuracy, sensitivity, and other issues relating to the reliability of abattoir-surveillance data for OJD in Australia, ${ }^{12}$ there is compelling evidence that there is considerable variation in OJD prevalence between and within states in Australia (socalled high-, medium-, and low-prevalence zones). Estimates of OJD prevalence derived at slaughter in 2011 were that of 2,117 known OJD-infected flocks (4.8\% of the national sheep flock), $60.1 \%$ of these flocks were located in NSW, $32.4 \%$ in Victoria, 30\% in Tasmania, 19.4\% each in South Australia and Western Australia, and none in Queensland. ${ }^{13}$

However, in areas of the national flock where vaccination has been occurring for 5 years or more, there have been significant declines in within-flock prevalence. A study in 2008-2009 of 37 OJD-infected flocks of varying initial OJD prevalence that had been vaccinated with Gudair for at least 5 years identified a decline in estimated prevaccination median prevalence of $2.72 \%$ to a postvaccination median prevalence of $0.72 \%$, using shedding-rate data from pooled fecal cultures (PFCs) for MAP. ${ }^{14}$ Prolonged pathological surveillance over 10 years of the tail of the mob from a high-prevalence OJD-infected farm that had been vaccinated diligently for a decade found that OJD cases with chronic granulomatous enterocolitis and mesenteric lymphadenitis and multibacillary lesions (so-called super shedders) declined from more than $50 \%$ of these suspected "ill sheep" within 4 years of the introduction of vaccine. Such cases currently occur annually at between $1.5 \%$ and $3 \%$ of sheep that are well below flock-average condition score at necropsy (personal observations).

\section{Change-management perspectives}

However, are these apparent positive changes in CLA and potentially OJD prevalence and producer concern mostly attributable to the introduction of widely available vaccination strategies for both diseases, and if not, what have been the other factors of relevance to these changes?

Changing the attitudes of farmers to improve animalhealth and welfare practices on the farm is challenging, particularly in encouraging the uptake and adoption of sustainable interventions that improve the management of disease risks. ${ }^{15}$ Change management refers to the understanding of how change is leveraged through strategy, structure, and operational mechanisms, as well as informal processes, including power, politics and conflict, culture, and leadership. ${ }^{16} \mathrm{~A}$ formal change-management perspective has rarely been applied to livestock disease management. However a recent example was the consideration of factors involved in improving the uptake and adoption of improved biosecurity for transboundary animal disease prevention by smallholding farmers. ${ }^{15}$ In the Mekong region of Southeast Asia, concerns have been raised that a vaccination-only policy for foot-and-mouth disease (FMD) may be unsustainable, due to the porous international borders and unregulated animal movements between countries at a time of increasing demand for meat by rapidly developing economies, with a change-management perspective providing a framework for reflection on the disease-control strategies required. ${ }^{15}$ A change-management perspective is also of relevance to a better understanding of the increasing global demand for improved animal welfare, and particularly pain management during aversive husbandry procedures. ${ }^{17}$ The impressive improvement in adoption of improved welfare practices on Australian sheep farms following the introduction of a farmer applied spray-on formulation (Tri-Solfen; Bayer Animal Health, Gordon, NSW, Australia) to manage the pain of the mulesing operation (used to create a bare area of the breech to reduce flystrike), has potential application to other invasive husbandry procedures. ${ }^{17}$ These examples of vaccines for CLA, OJD, FMD, and a pain-relief spray product suggest that empowering farmers to take ownership of managing 
animal disease problems is potentially a powerful extension tool for improving disease control and is highly relevant to considerations of animal health change management.

This paper examines the effectiveness of vaccination and disease-control extension programs and strategies used on farms and promoted by animal health authorities, and questions whether improved knowledge and attitudes towards CLA and OJD by sheep producers in Australia have been as important as the introduction of persistent vaccination programs. Change-management considerations are used to provide a framework for reflection on the factors involved in improving practices for CLA and OJD control, including drivers and motivation for change, resistance to change, knowledge management, farming system dimensions, and change-management leadership. Finally, it asks the question: does the diminution of CLA as a priority issue for the Australian sheep industry provide insights into the likely future of OJD management?

\section{Vaccination for CLA and OJD control}

After demonstration that the PLD exotoxin of CPTB could be used as a protective antigen, an effective toxoid vaccine for CLA was provided to Commonwealth Serum Laboratories (Melbourne, VIC, Australia) in 1978, and an optimal antigen dose and combination with clostridial components led to the release of Glanvac in 1984. ${ }^{1,11}$ A highly purified recombinant derivative of PLD was then shown to provide similar protection to that produced by the bacterin vaccine, but superiority in preventing the spread of infection beyond the site of inoculation plus efficacy of a combination of PLD antigen with five clostridial antigens in Australia led to the marketing and now-widespread use of " 6 in 1" vaccine. Field trials have shown variable rates of protection, from $25 \%$ to $90 \%$, following the vaccination of a previously unvaccinated infected flock with high challenge. Although vaccinating a flock will not prevent infection, it does reduce the number of sheep that develop lung lesions, and as older sheep are culled over time, the infectious challenge reduces, meaning fewer sheep will be infected. However, the vaccination program preferably consists of the administration of two doses at least 1 month apart and an annual booster a month before lambing or shearing. In commercial self-replacing wool flocks, it is most practical to administer the initial vaccine $6-8$ weeks after the start of the lambing season at lamb marking, with the second dose administered 4-5 weeks later at weaning. ${ }^{1}$

The control of MAP infection through hygiene management and the culling of test-positive animals has been suggested to have generally failed to produce the expected results, and thus a renewed focus on vaccination against this pathogen has been promoted as necessary. ${ }^{18} \mathrm{~A}$ meta-analysis run on the selected data on vaccination against MAP led to the conclusion that most studies reported that vaccination reduced risks of microbial contamination, reducing or delaying production losses and pathology. ${ }^{18}$ However vaccination did not fully prevent infection, with the majority of efficacious MAP vaccines considered as being rudimentary, with room for improvement in vaccine types and formulations. ${ }^{18}$ In Australia, OJD management under the National Johne's Disease Control Program is now dependent on widespread use of Gudair. This follows the success of vaccine field trials that commenced in 1999 under the National Ovine Johne's Disease Control and Evaluation Program (1998-2004) that are still continuing and have been recently reviewed. ${ }^{6}$

The initial study that led to the registration of Gudair (CZ Veterinaria, Porrino, Spain, imported into Australia by Pfizer Animal Health [now Zoetis]) examined the control of OJD by vaccination in merino sheep run under Australian pastoral conditions, using a single subcutaneous dose delivered behind the ear in lambs between 4 and 16 weeks of age. The study involved 600 vaccinated and 600 unvaccinated lambs on three farms experiencing significant OJD losses (5\%-15\% per annum), with sample collections conducted twice yearly until 4 or 5 years of age. ${ }^{19}$ Vaccination stimulated cell-mediated and humoral immune responses, reduced mortalities due to OJD by $90 \%$, and delayed and reduced fecal shedding of MAP. Although the amount of MAP excreted by the vaccinated groups was also reduced by at least $90 \%$ at most samplings, high levels of excretion by vaccinates occurred on some occasions, and all seven of the 600 vaccinates that died from OJD had multibacillary lesions. This indicates a risk that vaccinated sheep from OJD-infected properties may transmit OJD. Vaccine injection-site lesions were detected in almost $50 \%$ of sheep after 2 months, and persisted for at least 4 years in $20 \%-25 \%$ of vaccinates. ${ }^{19}$

Subsequent trials and field reports from Australia have attempted to understand more clearly the efficacy of Gudair. This is important for national disease control, but also because vaccination for paratuberculosis remains controversial in some parts of the world, largely based on concerns relating to the efficacy of the vaccine in decreasing the risk of infection and transmission of the organism, plus vaccine safety. ${ }^{6}$ The Australian experience has shown that vaccination with Gudair quickly eliminates the significant mortalities in OJD-infected, high-prevalence flocks. However, shedding of MAP from vaccinated sheep may persist for many years after 
commencing vaccination in an infected flock. ${ }^{20,21}$ Further, vaccination of all sheep was necessary, and the retention of unvaccinated mobs such as wethers (castrated males) on the farm was of high risk to recrudescence of MAP shedding. ${ }^{22}$ In a study of 37 flocks where vaccination of lambs had been applied for 6 years, shedding persisted in the majority of flocks, and a risk factor study in these flocks indicated probable associations with increased prevalence of OJD to include straying sheep, introduction of unvaccinated sheep into the flock, and the use of commercial contractors to do the vaccinations. ${ }^{6}$ Improvement of farm biosecurity and correct vaccination of all sheep in a flock were suggested as important management interventions to optimize the protection offered by vaccination. A recent study on Kangaroo Island (SA, Australia) indicated that where a majority of these practices are rigorously applied, eradication of the disease is potentially possible. ${ }^{6}$

Further studies on vaccine injection-site lesions in sheep caused by Gudair were conducted, confirming that this vaccine of a complete Freund's adjuvant nature, induced granulomatous cellulitis and lymphadenitis associated with oil droplets typical of "oil granulomata", and that these lesions occurring at and/or near the site of injection were common, but adverse consequences to vaccination were relatively uncommon. ${ }^{22,23}$ Importantly, deleterious outcomes did occur if the vaccine was incorrectly administered, as occurred with injection of vaccine into the dorsal cervical area, resulting in nonprogressive or progressive paralysis due to myonecrosis or granulomatous leptomeningitis, respectively (so-called OJD staggers), and production losses from injection in the maxilla or axilla of rams, or if flystrike occurred. These findings are not surprising, as dissection of tissues at necropsy of sheep vaccinated up to a week previously with Gudair placed in an intramuscular location revealed extensive movement of vaccine along the perimysium, with progressive necrosis and phagocytosis of muscle tissue in a $10 \mathrm{~cm}$ radius surrounding the site of injection (personal observations). Risk factors for adverse reactions included inadequate restraint of sheep, breed of sheep, expertise of the operator, poor injection technique, and inappropriate placement of vaccine. The study advocated increased attention to the proper restraint of animals, restricting vaccination to the recommended site behind the ear, careful placement of the vaccine into subcutaneous tissue to avoid drainage of vaccine material into such tissues as the spinal cord, and postvaccination supervision to address welfare concerns. ${ }^{22}$ Although vaccination-injection lesions were found at slaughter in $18 \%$ of adult sheep and $65 \%$ of lamb carcasses, no economic losses were incurred from the discounting of damaged carcasses, due to the extensive trimming that occurs at abattoirs. ${ }^{23}$

Unfortunately the Australian experience also included the recording of incidents of human exposure to the vaccine, with an incident rate of one per 7,406 vaccinations administered, including several where self-inoculation with Gudair vaccine required medical intervention. ${ }^{24}$ Both males and females and mostly hands, arms, feet, or legs have been involved. Most cases required extensive surgical removal of the injected vaccine to allow wound repair, often with open drainage and prolonged recovery times. Clearly, Gudair vaccine can cause prolonged granulomatous inflammation if inadvertently injected into human tissue. ${ }^{24}$ After self-inoculation, early surgical debridement of the damaged tissue and extensive drainage to remove the vaccine material is strongly advised to avoid progression to extensive necrosis. ${ }^{24,25}$

Despite the less than optimal efficacy of Gudair with prolonged MAP shedding by vaccinates, and the safety concerns for both humans and sheep, the use of vaccination to control paratuberculosis in Australia has been of enormous benefit to the sheep industry and to rural communities, and this needs to be recognized internationally. ${ }^{6}$ Australia is fortunate to be free of bovine tuberculosis, and the diagnostic test of choice for ovine paratuberculosis is PFC, so the presence of postvaccination serological responses is of minimal concern, except for animals being tested for export. Researchers worldwide continue to search for new vaccine candidates with improved efficacy and reduced negative side effects, including vaccines with less tissue-provoking adjuvants and potentially subunit vaccines that may have the advantage of not compromising diagnostic tests for either bovine tuberculosis or paratuberculosis. ${ }^{26}$ Although studies on subunit vaccines have demonstrated reduced fecal shedding, a lack of interference with immunodiagnostic assays for bovine tuberculosis and paratuberculosis, and less tissue provocation, it remains to be seen whether a product that can be registered for widespread use will prove to be more efficacious than Gudair.

\section{Extension programs for CLA and OJD control}

Control of CLA depends on vaccination in most countries, although the disease persists even after prolonged vaccination, consistent with the suppressive nature of CLA vaccination. ${ }^{1}$ As a 1990 study in Australia concluded that only 10\%-15\% of sheep producers were using the recommended CLA vaccination program, persistence of the infection on farms is perhaps not surprising. ${ }^{1}$ However, as this information was rapidly becoming historical, abattoir data from 2009 were examined, 
indicating further declines in CLA prevalence and provoking the reexamination of current vaccination practices on farms in NSW. The average CLA prevalence for New South Wales was $5.3 \%$, and within the three surveyed Livestock Health and Pest Authority regions (Tablelands, Central North and Central West) was $2.9 \%, 4.9 \%$, and $4.4 \%$, respectively. ${ }^{10}$ The attitude of the majority of producers surveyed in these three regions was that CLA was of little or no significance (75\%), but they were aware of the need for CLA control, with approximately 68\% using "6 in 1" vaccine and $39.9 \%$ as recommended, a significant improvement from the 1990 study. ${ }^{1}$ It was concluded that it was likely that the prolonged use of CLA vaccination had successfully contributed to reducing the prevalence of CLA across Australia and particularly in NSW. ${ }^{10}$ However, improvements in the communication of information on preventative management practices, the importance of using an approved vaccination program, and increasing producer awareness of the importance of CLA control were indicated. ${ }^{10}$

Control of OJD in Australia by regulatory, destocking, and other approaches was generally considered to have been a failure prior to the introduction of vaccination in 2002. OJD control by vaccination was then supplemented by the introduction of the "sheep health statement". This is a vendor-declaration system aimed at facilitating improved disease-risk awareness during the trading of sheep. Further, a program of biosecurity-risk awareness has commenced. ${ }^{27}$ It is very likely that the various iterations of the national OJD-control extension program have been important in improving awareness of the disease and its management, progressing from the National Ovine Johne's Disease Control and Evaluation Program 1998-2004, the National Approach to the Management of Ovine Johne's Disease in Australia 2004-2007, the OJD Management Plan 2007-2012, to the current OJD Management Plan 2013-2018, which commenced in July $2013 .^{27}$

\section{Change-management perspectives for CLA and OJD control Drivers and motivation for change}

Extrinsic (external) motivations are tangible rewards, and include money, status, and recognition from the peer group, although they may also involve punishment, being the threat of losing these tangibles. Intrinsic (internal) motivations are those derived from within, and involve intangible rewards, such as emotions, feelings of satisfaction, a sense of accomplishment or achievement, pride in one's work, or a desire to develop as much as possible to fulfill potential. ${ }^{28}$
Understanding motivations can assist in managing the changes in producer knowledge and attitudes (beliefs) that are often required to improve on-farm practices (behaviors). It is assessed by consideration of changes in knowledge, attitudes, and practices (KAP), and has been particularly useful in understanding change in the subsistence smallholder farming system to FMD. ${ }^{15,29}$ Changing farmer KAP is considered a more challenging process when invoking a disease risk-management strategy, such as a self-directed vaccination or biosecurity program, than a change in husbandry methods that results in direct increases in tangible rewards, such as financial returns from livestock that occur from improved nutrition. ${ }^{15}$ Drivers that motivate changes in KAP can be numerous, but mostly can be categorized as those that seek to avoid losses, such as strategies that address product integrity and reputation risks, and those that create socioeconomic incentives, such as opportunities for increasing returns.

Neither CLA nor OJD control have had clear drivers of a zoonotic or food-safety risk, although the debate on the potential role of MAP and thus paratuberculosis in Crohn's disease in humans persists with committed advocates and confirmed skeptics. ${ }^{30}$ Whether MAP is a causative agent in some cases of Crohn's disease or simply represents an incidental association remains a controversial topic. However current evidence suggests that the notion should not be so readily dismissed. ${ }^{30}$

CLA can infect numerous mammalian species, but by far the most significant are farmed small ruminants. ${ }^{1}$ It has been argued recently that the intense scrutiny of adult sheep carcasses for CLA has questionable benefit from a public health viewpoint, particularly as estimates of the impact of inspection for CLA on the density of indicator organisms on the surface of carcasses suggested that carcass hygiene could be improved if CLA inspection procedures were curtailed. ${ }^{31}$ However this may have the disadvantage of reducing the effectiveness of abattoir surveillance for estimates of prevalence for other diseases, including OJD.

There have been clear economic drivers that motivated government and sheep-industry agencies to support research that provides technical solutions, such as vaccines to address the financial impacts of both CLA and OJD in Australia. There have also been significant attempts to improve farmer KAP through state and national extension programs to aid their control. However, it is the social drivers for OJD control that have been particularly perplexing for the Australian sheep industry. Whereas CLA was historically considered largely a ubiquitous pathogen found through much of the sheep population, OJD emerged relatively 
recently in NSW and spread both insidiously and zonally, apparently infecting many properties some years before it was detected as a rapidly escalating problem of increasing mortalities and a sudden increase in the tail of the mob. Initially, NSW and then other state agencies used regulatory approaches to try to limit the spread of OJD. This was complicated by the early diagnostic tests being serological, with false positives and negatives creating significant anxiety and confusion until the testing regimen was replaced by the PFC. Unfortunately, a positive OJD diagnosis resulted in quarantine of the property, with almost no strategic options for release other than destocking for an extended period. Sadly, those that embraced the destocking strategy were generally reinfected due to the difficulties of identifying "clean" replacement stock, despite the early development of a market-assurance scheme to identify studs that were tested as unlikely to be infected. It is of historical importance to note that the regulatory strategy resulted in a profound social trauma, with infected studs and producers that relied on selling restocker animals losing their businesses, and widespread decreases in property values as sheep trading was prohibited from infected properties other than to slaughter. The regulatory program was abandoned in all states with the exception of South Australia, where there was low prevalence on the mainland (with the medium prevalence of Kangaroo Island managed intensively), and Queensland, where OJD has still not yet been diagnosed. The stigma of an OJD diagnosis led to division within rural communities, and for several years just prior to registration of the vaccine in 2002 , there was a tumultuous disturbance of community spirit, with a division between the OJD "haves" and the "have-nots", not assisted by those promulgating that OJD infection was largely the result of poor management. The provision of Gudair vaccine for use by farmers in Australia had an immediate impact, as it empowered sheep producers to achieve control of a sheepmortality situation that for many was escalating beyond their emotional resilience (personal observations).

\section{Resistance to change}

Sources of resistance to change by farmers are numerous, and a list of potential sources of resistance to biosecurity interventions to manage disease risks has recently been compiled. ${ }^{15}$ Social dynamics may be important when resistance is encountered, with low financial status, poor literacy, and limited knowledge of new technology producing embarrassment, loss of respect and anxiety, and invoking defense mechanisms, as discussed for OJD in Table 1. Prior to the introduction of Gudair vaccine, there was an apparent epidemic of personal anxiety in rural communities suffering from OJD losses that was so severe in NSW in 2000 that there were concerns that suicides may occur. One of the many responses of animal health authorities that occurred at that time was the introduction of the workshop program The Accidental Counsellor to equip rural extension workers with some basic emergency psychological skills.

A number of defense mechanisms invoked by farmers to sustain their belief in their ability to cope with the "OJD disaster" included:

1. Selective perception, where the farmer chooses not to accept a diagnosis or believe that OJD presents a significant threat, often claiming that poor-condition sheep have always occurred in Australia;

2. Scapegoating and conspiracy theories, where the farmer blames others, including neighbors or government services, for the inadvertent or willful introduction of OJD onto their farm or district, without embracing preventive practices, such as improving on-farm biosecurity;

3. Regression, where the farmer reverts to previous risky behaviors, such as the purchase of sheep from a known OJD-infected property, particularly if they have been informed the animals are vaccinated, believing that vaccinates are sufficiently protected despite extension messages to the contrary;

4. Denial, where the farmer chooses not to face the reality of the true risk or impact of OJD, believing that the losses only occur on farms that are poorly managed;

5. Displacement, where the farmer considers that OJD is caused by supernatural, divine, or spiritual influences rather than biological causes, particularly when faced with the uncertainties that naturally exist when a new disease is emerging and there is a lack of reliable information to enable definitive management strategies to be immediately promulgated.

Resistance to change by many farmers in Australia when reflecting on their disease status is also likely to result from cognitive bias, ${ }^{15}$ particularly considering the frequency and severity of severe weather events impacting on farming businesses. Bad weather may be blamed for high disease and low productivity outcomes, but positive outcomes are attributed to good management when favorable weather and other factors, such as rising demand and marketing influences, have contributed to improved farm profitability. A prolonged adverse weather event did commence in southeastern Australia in 2002 with the onset of one of the most severe droughts on record. ${ }^{7,8}$ This was accompanied by depressed wool prices from excessive supply of fine wool. These and other factors 
Table I Resistance and responses to ovine Johne's disease (OJD) change management on Australian sheep farms

Source of resistance
Loss of control of animal health
management: failure to act by embracing
self-determination.
Excessive uncertainty on disease risks:
rejection of advised intervention, as it is
costly and outside previous experience.

Surprises from imposed decisions: anxiety from lack of ownership of required interventions without sufficient time to prepare for the consequences.

Loss of reputation from positive diagnosis: failed businesses need acknowledgment that biosecurity practices have been insufficient.

Competence concerns: new information required to address disease risks, but basic understanding of disease model is lacking.

More work required: uncertainties best addressed by applied research and extension.

Ripple effects of changes required: leading to unexpected outcomes and push back from external sources.

Past resentments arise: change implementation required, but focus remains on scapegoating.

Threats from new technologies: replacing old strategies required.

\section{Leadership and behavioral} strategy required

Encourage participation and incorporate choice in decision making relating to vaccination and biosecurity, eg, continually promote OJD knowledge based on evidence. Admit uncertainties with disease-control strategies, but provide a clear vision and details of research aimed at providing clarity, with expected timelines, eg, applied research on OJD vaccine efficacy.

Creation and sharing of information that minimizes surprise plus time to adapt to interventions, including need to incorporate OJD vaccine in routine management, eg, evidence of persistent OJD vaccine use. Provision of strategies that address deficiencies of past practices and particularly failures of biosecurity, eg, promotion of more effective OJD biosecurity measures.

Positive reinforcement in clear language to ensure participants able to engage with new strategies, but not feel their skills obsolete, eg, implementing risk-based trading. Make requirements, standards, and benefits clear, rewarding pioneers, innovators, and supporters and identifying "champions", eg, enroll cooperators and early adopters in OJD field trials, surveys, field days, and workshops. Identify threats early and provide open discussion on expected impacts with all stakeholders, eg, risk-based trading requirements.

Acknowledgment of past mistakes required, but need to move forward promoted, eg, OJD regulatory failures and counseling. Avoid creating obvious losers, but also candor from the outset, eg, new diagnostic tests, vaccines, OJD-control strategies.
Example of successful farmer response

Farmers willing to seek diagnosis and obtain prevalence and control information to embrace interventions to improve farming system, including vaccination and biosecurity. Farmer communicates and engages with other stakeholders to foster an open dialogue, including visits to sites where interventions have been successfully embraced, and needs, concerns, and successes openly discussed.

Clear discussion or contribution to participatory research and extension on vaccine efficacy, with communication of expected results and benefits of interventions in public dialogue.

Provide a forum that enables "victims" to share their concerns and have skeptics' misinformation addressed with evidence-based information that remedies mistakes of the past, yet making it clear that the world has changed. Implement gradual change from old to new technologies, demonstrating commitment to provision of abundant information, education, training, mentoring, and support systems.

Link biosecurity to livestock production to ensure livelihood gains are clear, with champion farmers recruited to support promotion of appropriate interventions.

Identify new opportunities with a broader range of stakeholder groups outside initial boundaries and engage in open dialogue, providing support and assistance.

Recognize resentments of the past exist and letting their grievances be declared so as to enable their "moving on".

Offset losses with identification of opportunities, including promotion of lower disease risk as an efficiency gain for production and sustainable profitability.

Note: Adapted with permission Young JR, Evans-Kocinski S, Bush RD, Windsor PA. Improving smallholder farmer biosecurity in the Mekong region through change management. Transbound Emerg Dis. Epub November 8, $2013 .^{15} \odot 2013$ John Wiley \& Sons, Inc.

resulted in substantial structural changes in the Australian sheep industry, with a rapid decline in sheep numbers from approximately 105 million to 68 million in just a few years as producers destocked for drought management and moved to sheep meat, cross-breeding, or beef cattle enterprises. ${ }^{5}$

\section{Knowledge management}

Knowledge is critical in informing biosecurity interventions and investment decisions by farmers, with broader community support for change requiring very effective communication strategies, as occurred in the public awareness campaign to address disease-transmission risks during the eradication of
FMD in the Philippines a decade ago. ${ }^{32,33}$ Managing knowledge requires a conscious move from tacit to explicit knowledge (the subconscious to conscious). Tacit knowledge is highly personal and hard to formalize and articulate, so can be difficult to communicate, but in order for knowledge to invoke substantial change in biosecurity practices by the broader rural community, it needs to become explicit. ${ }^{15}$ In agricultural extension, field days and "cross-visits" that enable access and close communication to "champion" or "early adopter" farmers have been shown to be a powerful means of assisting tacit knowledge transfer. ${ }^{29}$ This approach proved to be important in NSW in the early OJD "crisis", where several farmers that had 
recently suffered severe losses volunteered to speak in public forums about their experiences with the disease and use of Gudair vaccination. The traditional rural press (newspaper, radio, and television) has had an important ongoing role in providing a public forum for the OJD debate. However, as with many scientific debates, considerable misinformation on OJD has been promulgated by the rural media, and the ample space provided to non-evidence-based views of skeptics and the antiscience lobby has impacted negatively on OJD program policy development (personal observations). However newer communication opportunities using electronic social media, including "webinars", ${ }^{34}$ newsletters,,${ }^{35}$ and blogs, ${ }^{36}$ have emerged to support OJD knowledge management, assisting efforts needed to address misinformation. These recent developments are proving to be very successful in transmitting learning on OJD from the established "endemic" areas to producers and their advisors in those areas where the disease has emerged more recently.

It is doubtful whether widespread knowledge of CLA control has been achieved in the Australian sheep industry, particularly as in several surveys farmers have demonstrated that explicit knowledge of the disease is weak. ${ }^{9,10}$ It is also unlikely that with continued widespread usage of "6 in 1" vaccine, broader community efforts to address CLA knowledge deficits will not be of sufficient priority in Australia, despite the clearly identified knowledge deficits that currently exist. ${ }^{10}$ However, addressing the knowledge deficits on problem farms that are repeat offenders with CLA lesions at abattoirs is advisable to protect the reputation of the substantial export sheep-meat market.

Although peer-reviewed published surveys of OJD knowledge and practices in the Australian rural community are lacking, there has been considerable effort by many government and stakeholder agencies to engage sheep producers in OJD control, as inferred earlier. Multitudes of workshops, field days, meetings, and forums around the country have encouraged the prolonged use of Gudair vaccine, improvements in on-farm biosecurity, and adoption of disease-risk awareness through the risk-based vendor-declaration trading mechanism. ${ }^{27}$ Of interest are statements by numerous NSW sheep producers following the relatively sudden emergence of the "OJD crisis", provoking reflection on general health management of their flocks. Many owners of OJD-infected flocks suffering significant mortalities observed that Gudair vaccination rapidly resolved their losses, and this apparently motivated efforts to adopt or improve other "best practice" animal health strategies, including CLA control, through a more rigorous vaccination program. ${ }^{1}$
That Gudair vaccine had to be delivered far more carefully than "6 in 1" required producers also to examine their vaccination techniques, both to protect themselves and their families or staff from self-harm and to minimize adverse reactions in sheep. Some producers also observed that the injection lesions in sheep following Gudair vaccination for OJD closely resemble CLA lesions, causing initial concerns regarding the diagnosis of superficial suppurative or granulomatous lesions ${ }^{1}$ and even unsubstantiated claims that CLA vaccination may protect against OJD. Further, the occasional occurrence of falsepositive reactors to OJD on serologic tests in consignments of small ruminants for export has been attributed to potential serologic cross-reaction to CLA vaccination, confirming the importance of knowledge of CLA-control procedures when preparing animals for export. For these reasons, it is appropriate that careful consideration of OJD and CLA control by vaccination and extension programs is reviewed.

\section{Farming system dimensions}

A farming system is an organized collection of production components, including inputs and outputs that are integrated ${ }^{15}$ to preferably accomplish optimal profitability and sustainability, with each component providing ongoing feedback to the other components. Australian sheep-farm inputs include financial capital, labor, land and water resources including pastures, fertilizers, crops, feed reserves, farm sheds and equipment including vehicles, and importantly, the livestock, various advisory and product/service providers, and animal health products. Sheep-farm outputs are tangible saleable products emerging from the system, such as the annual wool payment and sale of "cull for age" sheep, plus additional benefits, including hopefully an improving quality of life, pride in achievement, and social standing from peer recognition. Failures within the system generally impact on the other components of the system, as observed when OJD losses cause a measurable decline in farm income, ${ }^{7,8}$ force a major change in enterprise selection, ${ }^{5}$ or result in emotional stress and negative social consequences, as described. Effective extension programs require an understanding of the complexity of the farming system. They should recognize that animal health decisions by producers, such as adoption of a vaccination or biosecurity program for CLA and/or OJD, are not made in isolation from broader farm-management considerations, and are frequently compromised by more pressing priorities. Increasingly, tools that assist farmers in making investment decisions within their farming system have become available, including a program that examines the break-even point of investing in Gudair vaccine for OJD control. ${ }^{37}$ 


\section{Change-management leadership}

The legal responsibility for managing animal health in Australia has historically and still remains a state legislative responsibility. Cooperative arrangements between the state departments of agriculture or primary industries have traditionally been negotiated on an as-needs basis in consultation with the Commonwealth (federal) Government Department of Agriculture that is primarily responsible for international issues. This arrangement worked well for many years, with some notable successes including eradication of bovine pleuropneumonia, tuberculosis, and brucellosis, plus avian influenza on numerous occasions. This was the situation with the initial studies on CLA, where informal relationships between researchers from different states enabled the collation of a publishable national perspective, despite major differences between priorities, resources, and operations between the states. ${ }^{9}$

However, there was a long-held concern that the costsharing arrangements between the multiple jurisdictions for the eradication of FMD or a similar transboundary disease causing a major national emergency that would severely disrupt the export-dependent livestock industries could come under significant pressure. This led to the development in 1996 of the not-for-profit company Animal Health Australia (AHA) to facilitate responsive management and coleadership of national animal health issues on behalf of a broad range of stakeholders. AHA now manages more than 50 national programs that improve animal and associated human health, biosecurity, market access, livestock welfare, productivity, and food safety and quality. ${ }^{38}$ The "national animal health system" is a broad informal description of the wide range of government, commercial, and individual interests that benefit from Australia's favorable animal health status, with AHA aiming to create collaborative partnerships through programs and projects that share this common objective. ${ }^{38}$ AHA assisted coordination of the state abattoir-surveillance programs that provided recent prevalence data on CLA and OJD, as presented. However OJD control has proved to be a most challenging task, involving prolonged negotiations with governments, industry, and other stakeholders in developing the series of national OJD-control programs, as discussed. Unfortunately, the latest iteration of the OJD national program, being the OJD Management Plan 2013-2018 that commenced in July 2013, ${ }^{38}$ was accompanied by a major campaign led by a disaffected producer group that attempted to derail the program. A major objection was the proposal to introduce regional biosecurity-management plans that would formally discourage the trading of sheep from OJD high- and medium-prevalence zones to low-prevalence zones. Their motivation appeared to be driven by a desire to facilitate the trading of sheep that were vaccinated, in the belief they posed little risk of disease transmission. ${ }^{35}$ This resulted in an organized attack on the credibility of the science that established that vaccinates from OJD-infected properties posed a risk of disease transmission. Although a compromise between the stakeholder groups was achieved that enabled the program to proceed, the removal of zoning from the current national plan has potentially diminished the effectiveness of extension efforts that are intended to illustrate the clearly established differences in OJD prevalence and thus risk of trading across the country.

\section{Conclusion}

Consideration of change-management factors relevant to CLA and OJD control provides insights into the controversies that have emerged in attempts to control these diseases, particularly OJD. However, does the diminution of CLA as a priority issue for the Australian sheep industry provide insights into the likely future for OJD management in Australia? Inclusion of CLA vaccine with clostridial vaccine components enabled widespread adoption of routine and thus persistent CLA vaccination across a substantial proportion of the national flock, resulting in significant reductions in CLA in the national flock, estimated from abattoirsurveillance data as having declined from $26 \%$ in 1995 to $5.2 \%$ in 2009. Although there has been improved adoption of the recommended CLA vaccination program, the low level of recognition of the importance of CLA suggests that it is more likely that the innovation of a convenient means of administering the vaccine (as with "6 in 1") has had the most significant impact on the prevalence of CLA.

Evaluating the use of Gudair vaccine is problematic, as a multicomponent vaccine is not a strategy that is appropriate for the currently available OJD vaccines. The series of national OJD-control and -management programs that have encouraged a number of interventions, including the persistent use of vaccination in flocks infected with OJD, identified as necessary from prolonged research on field-vaccine efficacy, have clearly been important. Recent estimates of prevaccination median prevalence of $2.72 \%$ declining to a postvaccination median prevalence of $0.72 \%$ over 5 years or more of using the vaccine is encouraging. However the national OJD-management program was significantly challenged in 2013, with parts of the sheep industry not willing to accept the research message that vaccinates from OJDinfected properties remain a risk for disease transmission 
if traded. This indicates the great need for the scientific community to actively reinforce evidence-based extension messages to ensure the viability of national disease-control programs, as is now occurring with vaccination for childhood diseases. It has become increasingly important to confront the skeptics with evidence-based research that counters commercial and personal bias priorities that ignore population disease-transmission risk. Strong leadership by health professionals in national programs has been shown to be essential, as has a willingness to engage the majority of stakeholders in a consultative process.

There is cautious optimism emerging that the significant reductions in within-flock OJD prevalence in vaccinated flocks over the past decade, plus promotion of improved biosecurity through risk-based trading and on-farm biosecurity programs in addition to persistent vaccination, will have a major impact, and signal the trend for the future of OJD in Australia. However, encouraging producers to persist with vaccination despite the apparent absence of clinical OJD remains challenging, especially without a convenient vaccine-administration system, as exists for CLA. A deeper understanding of the dynamics of these chronic infections and the role of vaccination as a suppressant of subclinical OJD is required by the rural community. Importantly, it needs to be recognized globally that the positive impact on disease control of persistent vaccination over many sheep generations in flocks infected with CLA and/or OJD is generally not well understood outside Australia. Despite concerns of vaccine efficacy and issues with their administration, vaccination strategies for both diseases have had profoundly positive impacts on the health of Australian sheep and the welfare of sheep producers.

\section{Acknowledgments}

The collaborations of many colleagues over a considerable number of years was integral to this paper, and in particular Drs James Young, Russell Bush, and Clare Phythian provided valuable feedback on the manuscript. Several of the studies discussed in this review were funded by sheep-industry agencies, and their contributions are gratefully acknowledged in papers sourced for this review from our research team.

\section{Disclosure}

The author reports no conflicts of interest in this work.

\section{References}

1. Windsor PA. Control of caseous lymphadenitis. Vet Clin North Am Food Anim Pract. 2011;27:198-202.

2. Windsor PA. Research into vaccination against ovine Johne's disease in Australia. Small Rumin Res. 2006;62:139-142.
3. Lambeth C, Reddacliff LA, Windsor PA, Abbott KA, McGregor H, Whittington RJ. Intrauterine and transmammary transmission of Mycobacterium avium subsp paratuberculosis in sheep. Aust Vet J. 2004;82: 504-508.

4. Whittington RJ, Windsor PA. In utero infection with Mycobacterium avium subsp. paratuberculosis: a critical review and meta-analysis. Vet J. 2009;179:60-69.

5. Meat and Livestock Australia. Australia's sheepmeat industry. 2013. Available from: http://www.mla.com.au/Cattle-sheep-and-goatindustries/Industry-overview/Sheep. Accessed December 11, 2013.

6. Windsor PA. Understanding the efficacy of vaccination in controlling ovine paratuberculosis. Small Rumin Res. 2013;110:161-164.

7. Bush RD, Windsor PA, Toribio JA. Losses of adult sheep due to ovine Johne's disease in 12 infected flocks over a 3-year period. Aust Vet J. 2006; $84: 246-253$.

8. Bush RD, Toribio JA, Windsor PA. The impact of malnutrition and other causes of losses of adult sheep in 12 flocks during drought. Aust Vet J. 2006;84:254-260.

9. Paton M, Walker S, Rose I, Watt G. Prevalence of caseous lymphadenitis and usage of caseous lymphadenitis vaccines in sheep flocks. Aust Vet $J$. 2003;81:91-95.

10. Bush RD, Barnett R, Links I, Windsor PA. Using abattoir surveillance and producer surveys to investigate the prevalence and current preventative management of caseous lymphadenitis in merino flocks in Australia. Anim Prod Sci. 2012;52:675-679.

11. Baird GJ, Fontaine MC. Corynebacterium pseudotuberculosis and its role in ovine caseous lymphadenitis. J Comp Pathol. 2007;137:179-210.

12. Bradley TL, Cannon RM. Determining the sensitivity of abattoir surveillance for ovine Johne's disease. Aust Vet J. 2008;83:633-636.

13. Animal Health Australia. JD News. Summer 2012. Available from: http://www.animalhealthaustralia.com.au/wp-content/uploads/2011/05/ JD-News-Summer-2012.pdf. Accessed December 11th, 2013.

14. Dhand NK, Johnson W, Eppleston J, Windsor PA. Comparison of preand post-vaccination ovine Johne's disease prevalence by incorporating information from multiple sources using Bayesian methods. Prev Vet Med. 2013;111:81-91.

15. Young JR, Evans-Kocinski S, Bush RD, Windsor PA. Improving smallholder farmer biosecurity in the Mekong region through change management. Transbound Emerg Dis. Epub November 8, 2013.

16. Senior B, Swailes S. Organizational Change. 4th ed. Upper Saddle River (NJ): Prentice Hall; 2010.

17. Windsor PA, Lomax S. Addressing welfare concerns regarding control of cutaneous myiosis in Australia. Small Rumin Res. 2013;110: $165-169$.

18. Bastida F, Juste RA. Paratuberculosis control: a review with a focus on vaccination. J Immune Based Ther Vaccines. 2011;9:1-17.

19. Reddacliff LA, Eppleston J, Windsor PA, Whittington RJ, Jones S. Efficacy of a killed vaccine for the control of paratuberculosis in Australian sheep flocks. Vet Microbiol. 2006;115:77-79.

20. Eppleston J, Reddacliff L, Windsor PA, Links I, Whittington R. Preliminary observations on the prevalence of sheep shedding Mycobacterium avium subsp. paratuberculosis after 3 years of a vaccination program for ovine Johne's disease (OJD). Aust Vet J. 2005;83:611-612.

21. Eppleston J, Windsor PA, Whittington R. Impact of unvaccinated merino wether lambs on shedding of Mycobacterium avium subspecies paratuberculosis in flocks vaccinating for ovine Johne's disease. Aust Vet J. 2010;89:38-40.

22. Windsor PA, Eppleston J. Lesions in sheep following administration of a vaccine of a Freund's complete adjuvant nature used in the control of ovine paratuberculosis. N Z Vet J. 2006;54:237-241.

23. Eppleston J, Windsor PA. Lesions attributed to vaccination of sheep with Gudair for the control of ovine paratuberculosis: post-farm economic impacts at slaughter. Aust Vet J. 2007;85:129-133.

24. Windsor PA, Bush RD, Links I, Eppleston J. Injury caused by selfinoculation with a vaccine of a Freund's complete adjuvant nature (Gudair) used for control of ovine paratuberculosis. Aust Vet J. 2005;83: 216-220. 
25. Richardson G, Links I, Windsor P. Gudair (OJD) self-inoculation: a case for early debridement. Med J Aust. 2005;183:151-152.

26. Patton EA. Paratuberculosis vaccination. Vet Clin North Am Food Anim Pract. 2011;27:573-580.

27. Animal Health Australia. Ovine Johne's disease in Australia: national OJD management plan 2013-2018. 2013. Available from: http://www. animalhealthaustralia.com.au/programs/johnes-disease/ovine-johnesdisease-in-australia. Accessed December 11, 2013.

28. Evans-Kocinski S. What It Takes, Personal Development Strategies for the Office. Melbourne: Pitman; 1992.

29. Nampanya S, Sothoeun S, Rast L, Windsor PA. Improvement of farmer knowledge of production, health and biosecurity between 2008 and 2010 in Cambodia. Transbound Emerg Dis. 2012;59:117-127.

30. Chiodini RJ, Chamberlin WM, Sarosiek J, McCallum RW. Crohn's disease and the mycobacterioses: a quarter century later. Causation or simple association? Crit Rev Microbiol. 2012;38:52-93.

31. Jordan D, Sentance CB, Spooncer WF, Balan JA, Morris SM. Inspection of lymph nodes for caseous lymphadenitis and its effect on the density of microbes on sheep carcasses. Meat Sci. 2012;92:837-840.

32. Windsor PA. Review: perspectives on Australian animal health aid projects. Transbound Emerg Dis. 2011;58:375-386.
33. Windsor PA, Freeman PG, Abila R, et al. Control and eradication of foot and mouth disease in the Bicol Surveillance Buffer Zone in the Philippines. Transbound Emerg Dis. 2011;58:421-433.

34. Animal Health Australia. Ovine Johne's disease in Australia: Animal Health Australia webinar on OJD vaccination. Available from: http:// record.redbackconferencing.com.au/Recordings/Webinar/AHA_19_ DEC_1800.wmv. Accessed December 12, 2013.

35. Walker W. Attempting the near impossible: understanding the control of OJD! Classings Cullings'13. 2013. Available from: http://www. classings.com.au/media/newsletter/classingscullingsnewsletter2013lowres.pdf. Accessed December 12, 2013.

36. Walker W. Bill's blog. Available from: http://www.classings.com.au/ pages/posts/letrsquos-take-the-axe-to-6.php. Accessed December 12, 2013.

37. Bush RD, Windsor PA, Toribio JA, Webster SR. Financial modelling of the potential cost of ovine Johne's disease and benefit of vaccination in southern New South Wales. Aust Vet J. 2008;86:398-403.

38. Animal Health Australia. Strategic Plan 2010-2015: Working Together for Animal Health. Available from: http:/www.animalhealthaustralia. com.au/wp-content/uploads/2011/05/AHA-Strategic-Plan-2010-2015. pdf. Accessed January 28, 2014.
Veterinary Medicine: Research and Reports

\section{Publish your work in this journal}

Veterinary Medicine: Research and Reports is an international, peer-reviewed, open access journal publishing original research, case reports, editorials, reviews and commentaries on all areas of veterinary medicine. The manuscript management system is completely online and includes a very quick and fair peer-review system.

\section{Dovepress}

Visit http://www.dovepress.com/testimonials.php to read real quotes from published authors. 